\title{
Countably Many Positive Solutions for Nonlinear Singular n-Point Boundary Value Problems
}

\author{
Zhenguo Li, Youlin Shang* \\ Department of Mathematics and Statistics, Henan University of Science and Technology, Luoyang, China \\ Email: ${ }^{\text {mathshang@sina.com }}$
}

Received 18 May 2014; revised 22 June 2014; accepted 5 July 2014

Copyright (C) 2014 by authors and Scientific Research Publishing Inc.

This work is licensed under the Creative Commons Attribution International License (CC BY). http://creativecommons.org/licenses/by/4.0/

(c) (i) Open Access

\begin{abstract}
In this paper, a fixed-point theorem has been used to investigate the existence of countable positive solutions of $n$-point boundary value problem. As an application, we also give an example to demonstrate our results.
\end{abstract}

Keywords

Boundary Value Problem, Existence of Positive Solutions, Fixed-Point Theorem

\section{Introduction}

The multi-point boundary value problems arising from applied mathematics and physics have received a great deal of attention in the literature (for instance, [1]-[4] and references therein). But, by so far, few results are about the existence of more than five solutions. To the author's knowledge, there are very few papers concerned with the existence of countable positive solutions for multiple point BVPS (for instance, [5] and references therein). In [5], the authors discussed the existence of countable positive solutions of $n$-point boundary value problems for a $p$-Laplace operator on the half-line. Directly inspired by [5], in this paper, by using a fixed-point theorem, we study the existence of countable positive solutions of the following $n$-point boundary value problems.

$$
\begin{array}{lr}
u^{\prime \prime}(t)+a(t) f(u(t))=0, & t \in(0,1), \\
u^{\prime}(0)=0, & u(1)=\sum_{i=1}^{n-2} a_{i} u\left(\xi_{i}\right),
\end{array}
$$

${ }^{*}$ Corresponding author. 
where $a_{i} \in[0,+\infty), \quad 0<\sum_{i=1}^{n-2} a_{i}<1, \quad 0<\xi_{1}<\xi_{2}<\cdots<\xi_{n-2}<1, \quad f \in C([0,+\infty),[0,+\infty))$.

$a(t):[0,1] \rightarrow[0,+\infty)$ and $a(t) \not \equiv 0$ has countable many singularities in $\left[0, \frac{1}{2}\right)$.

This kind of problem arises in the study of a number of chemotherapy, population dynamics, ecology, industrial robotics and physics phenomena. Moreover, many problems in optimal control system, neural network (for example in BAM neural network) and information systems for computational science and engineering (especially in Internet-based computing) can be established as differential equation models with boundary condition (see, for instance, [6] and references therein).

At the end of this section, we state some definitions and lemmas which will be used in Section 2 and Section 3.

Definition 1.1 A map $\alpha$ is said to be a nonnegative, continuous, concave function on a cone $P$ of a real Banach space $E$, if $\alpha: P \rightarrow[0,+\infty)$ is continuous, and

$$
\alpha(t x+(1-t) y) \geq t \alpha(x)+(1-t) \alpha(y)
$$

for all $x, y \in P$ and $t \in[0,1]$.

Definition 1.2 Given a nonnegative continuous function $\gamma$ on a cone $P \subset E$, for each $d>0$, we define the set $P(\gamma, d)=\{x \in P: \quad \gamma(x)<d\}$

Lemma 1.1 [7] Let $E$ be a Banach space and $P \subset E$ be a cone in $E$. Let $\alpha, \beta, \gamma$, be three increasing, nonnegative and continuous functions on $P$, satisfying for some $c>0$ and $M>0$ such that

$$
\gamma(x) \leq \beta(x) \leq \alpha(x), \quad\|x\| \leq M \gamma(x)
$$

for all $x \in \overline{P(\gamma, c)}$. Suppose that there exists a completely continuous operator $T: \overline{P(\gamma, c)} \rightarrow P$ and $0<a<b<c$ such that

1) $\gamma(T x)<c$, for $x \in \partial P(\gamma, c)$.

2) $\beta(T x)>b$, for $x \in \partial P(\beta, b)$.

3) $P(\alpha, a) \neq \varnothing$, and $\alpha(T x)<a$, for $x \in \partial P(\alpha, a)$.

Then $T$ has at least three fixed points $x_{1}, x_{2}, x_{3} \in P(\gamma, c)$ such that

$$
0 \leq \alpha\left(x_{1}\right)<a<\alpha\left(x_{2}\right), \quad \beta\left(x_{2}\right)<b<\beta\left(x_{3}\right), \quad \gamma\left(x_{3}\right)<c .
$$

This paper is organized as follows: The preliminary lemmas are in Section 2. The main results are given in Section 3. Finally, in Section 4, we give an example to demonstrate our results.

\section{The Preliminary Lemmas}

In this paper, we will use the following space $E=C[0,1]$ and $E$ is a Banach space with the norm$\|u\|=\sup _{t \in[0,1]}|u(t)|$. Let $J=[0,1]$, we define a cone $K \subset E$ by

$$
K=\{u \in E: u(t) \text { is a non-increasing and nonnegative concave function on } J\} .
$$

For convenience, let us list some conditions.

$\left(H_{1}\right) f \in C([0,+\infty),[0,+\infty))$ and on any subinterval of $J$ and when $u$ is bounded, $f(u(t))$ is bounded on $J$.

$\left(H_{2}\right)$ There exists a sequence $\left\{t_{i}\right\}_{i=1}^{\infty}$ such that $t_{i+1}<t_{i}, t_{1}<\frac{1}{2}, \lim _{i \rightarrow \infty} t_{i}=t_{0}, \lim _{t \rightarrow t_{i}} a(t)=\infty, \quad i=1,2, \cdots$, and $\int_{0}^{+\infty} a(t) \mathrm{d} t<+\infty$.

Lemma 2.1. Let $0<\left(1-\sum_{i=1}^{n-2} a_{i}\right)<0, h(t) \in C[0,1]$ and $h(t) \geq 0$ on $(0,1)$, then the boundary value problem

$$
u^{\prime \prime}(t)+h(u(t))=0, \quad t \in(0,1),
$$




$$
u^{\prime}(0)=0, \quad u(1)=\sum_{i=1}^{n-2} a_{i} u\left(\xi_{i}\right)
$$

has a unique solution

$$
u(t)=-\int_{0}^{t}(t-s) h(u(s)) \mathrm{d} s+\frac{1}{1-\sum_{i=1}^{n-2} a_{i}} \int_{0}^{1}(t-s) h(u(s)) \mathrm{d} s-\sum_{i=1}^{n-2} a_{i} \int_{0}^{\xi_{i}}\left(\xi_{i}-s\right) h(u(s)) \mathrm{d} s
$$

Proof. The proof is easy, so we omit it.

By $u^{\prime}(t)=-\int_{0}^{t} h(u(s)) \mathrm{d} s \leq 0, u^{\prime \prime}(t)=-h(u(s)) \leq 0$, we know $u(t)$ is decreasing and concave on $[0,1]$. Then we have

$$
\begin{gathered}
\max _{t \in[0,1]} u(t)=u(0)=\frac{\int_{0}^{1}(1-s) h(u(s)) \mathrm{d} s-\sum_{i=1}^{n-2} a_{i} \int_{0}^{\xi_{i}}\left(\xi_{i}-s\right) h(u(s)) \mathrm{d} s}{1-\sum_{i=1}^{n-2} a_{i}} \leq \frac{\int_{0}^{1} h(u(s)) \mathrm{d} s}{1-\sum_{i=1}^{n-2} a_{i}} \\
\max _{t \in[0,1]} u(t)=u(1)=\frac{1}{1-\sum_{i=1}^{n-2} a_{i}}\left(\sum_{i=1}^{n-2} a_{i} \int_{\xi_{i}}^{1}(1-s) h(u(s)) \mathrm{d} s+\sum_{i=1}^{n-2} a_{i} \int_{0}^{\xi_{i}}\left(1-\xi_{i}\right) h(u(s)) \mathrm{d} s\right) \geq 0
\end{gathered}
$$

From (2.3), (2.4) and the concavity of $u(t)$, we can easily get the following lemma.

Lemma 2.2. Let $0<\left(1-\sum_{i=1}^{n-2} a_{i}\right)<0$, if $h(t) \in C[0,1]$ and $h(t) \geq 0$ on $C[0,1]$, then the unique solution $u(t)$ of (2.1)-(2.2) satisfies $u(t) \geq 0, t \in[0,1]$ and $\inf _{0 \leq t \leq 1} u(t) \geq \lambda\|u\|$, where $\lambda=\frac{\sum_{i=1}^{n-2} k_{i}\left(1-\xi_{i}\right)}{1-\sum_{i=1}^{n-2} k_{i} \xi_{i}}<1$.

For $u \in K$, we define an operator $A: K \rightarrow E$ by

$$
(A u)(t)=-\int_{0}^{t}(t-s) a(s) f(u(s)) \mathrm{d} s+\frac{1}{1-\sum_{i=1}^{n-2} a_{i}}\left(\int_{0}^{1}(1-s) a(s) f(u(s)) \mathrm{d} s-\sum_{i=1}^{n-2} a_{i} \int_{0}^{\xi_{i}}\left(\xi_{i}-s\right) a(s) f(u(s)) \mathrm{d} s\right)
$$

For $u \in K$, then $u \in E$, $\sup _{t \in J}|u(t)|<+\infty$, by $\left(H_{1}\right)$, we know $f(u(t))$ is bounded on $J$.

So there exists $M_{0} \geq 0$, such that

$$
\max _{s \in J} f(u(s)) \leq M_{0} .
$$
is

It is easy to see that $(A u)(t)$ is decreasing and concave on $[0,1]$. Then for $u \in K$, we have $A u \in K$, that

$$
A K \subset K .
$$

From $\left(H_{2}\right)$, (2.3) and (2.6), we have

$$
\max _{t \in[0,1]}(A u)(t)=(A u)(0) \leq \frac{M_{0} \int_{0}^{1} a(s) \mathrm{d} s}{1-\sum_{i=1}^{n-2} a_{i}}<+\infty .
$$

From (2.7), (2.8), we can get the following lemma.

Lemma 2.3. Suppose $\left(H_{1}\right)$ and $\left(H_{2}\right)$ are satisfied. Then $A: K \rightarrow K$ is bounded.

Lemma 2.4. Assume $\left(H_{1}\right),\left(H_{2}\right)$ are satisfied, then $A: K \rightarrow K$ is completely continuous.

Proof. From Lemma 2.2, we know $A$ is bounded. If $W \in K$ is a bounded subset of $K$, then $A W$ is uniformly bounded on $I=[0,1]$. 
For any $u \in W, t_{1}, t_{2} \in[0,1]$, without loss generality, we may assume $t_{2}>t_{1}$, by (2.5), (2.6), $\left(H_{2}\right)$, we have

$$
\begin{aligned}
\left|(A u)\left(t_{1}\right)-(A u)\left(t_{2}\right)\right| & =\left|\int_{0}^{t_{1}}\left(t_{1}-s\right) a(s) f(u(s)) \mathrm{d} s-\int_{0}^{t_{2}}\left(t_{2}-s\right) a(s) f(u(s)) \mathrm{d} s\right| \\
& \leq\left|\int_{t_{1}}^{t_{2}} t_{2} a(s) f(u(s)) \mathrm{d} s\right|+\left|\left(t_{2}-t_{1}\right) \int_{0}^{t_{1}} a(s) f(u(s)) \mathrm{d} s\right|+\left|\int_{t_{1}}^{t_{2}} s a(s) f(u(s)) \mathrm{d} s\right| \rightarrow 0,
\end{aligned}
$$

uniformly as $t_{1} \rightarrow t_{2}$.

So $A W$ is equi-continuous on $I=[0,1]$.

At last, by (2.5), $\left(\mathrm{H}_{2}\right)$, the Lebesgue dominated convergence theorem and continuity of $f$, we know $A$ is continuous. Then by the Arzela-Ascoli theorem, we can get that $A: K \rightarrow K$ is completely continuous.

\section{Main Results}

Let $\theta_{k} \in\left(t_{k}, t_{k+1}\right), \theta_{k}<r_{k}<1-\theta_{k}$ and $\gamma_{k}, \beta_{k}, \alpha_{k}$ be three nonnegative, decreasing and continuous functions with

$$
\gamma_{k}(u)=\max _{r_{k} \leq t \leq 1-\theta_{k}} u(t), \quad \beta_{k}(u)=\min _{\theta_{k} \leq t \leq r_{k}} u(t), \quad \alpha_{k}(u)=\max _{\theta_{k} \leq t \leq 1-\theta_{k}} u(t) .
$$

Obviously, for $\forall u \in K$ we have $\gamma_{k}(u) \leq \beta_{k}(u) \leq \alpha_{k}(u)$.

In the following, we let

$$
\rho=\left(1-\sum_{i=1}^{n-2} a_{i}\right)^{-1} \int_{0}^{1} a(s) \mathrm{d} s, \quad \eta=\left(1-\sum_{i=1}^{n-2} a_{i}\right)^{-1}\left(\sum_{i=1}^{n-2} a_{i} \int_{\xi_{i}}^{1}(1-s) a(s) \mathrm{d} s\right)
$$

Then it is easy to see $\rho>\eta$.

The main result of this paper is as follows.

Theorem 3.1. Assume that $\left(H_{1}\right)-\left(H_{2}\right)$ hold. Let $\left\{\theta_{k}\right\}_{k=1}^{\infty}$ be such that $\theta_{k} \in\left(t_{k}, t_{k+1}\right)$

$(k=1,2, \cdots),\left\{a_{k}\right\}_{k=1}^{\infty},\left\{b_{k}\right\}_{k=1}^{\infty}$ and $\left\{c_{k}\right\}_{k=1}^{\infty}$ be such that $c_{k+1}<a_{k}<\lambda b_{k}<c_{k}$ and $\rho b_{k}<\eta c_{k} .(k=1,2, \cdots)$.

Furthermore for each natural number $k$ we assume that $f$ satisfies:

$\left(H_{3}\right) f(u)<\frac{c_{k}}{\rho}$ for all $0 \leq u(t) \leq c_{k} / \lambda$;

$\left(H_{4}\right) f(u)<\frac{b_{k}}{\eta}$ for all $b_{k} \leq u(t) \leq b_{k} / \lambda$

$\left(H_{5}\right) f(u)<\frac{a_{k}}{\rho}$ for all $0 \leq u(t) \leq a_{k} / \lambda$.

Then the BVP (1.1)-(1.2) has at least three infinite families of positive solutions $\left\{u_{1 k}\right\}_{k=1}^{\infty},\left\{u_{2 k}\right\}_{k=1}^{\infty}$ and $\left\{u_{3 k}\right\}_{k=1}^{\infty}$ with

$$
0 \leq \alpha_{k}\left(u_{1 k}\right)<a_{k}<\alpha_{k}\left(u_{2 k}\right), \quad \beta_{k}\left(u_{2 k}\right)<b_{k}<\beta_{k}\left(u_{3 k}\right), \quad \gamma_{k}\left(u_{3 k}\right)<c_{k} \text {, for } k \in N .
$$

Proof. From the definition of $A$, (2.7) and Lemma 2.4, it is easy to see that $A: \overline{K\left(\gamma_{k}, c_{k}\right)} \rightarrow K$, for $k \in N$ is completely continuous.

Next we show all the conditions of Lemma 1.2 hold.

For any $u \in K$, it is easy to see $\gamma_{k}(u) \leq \beta_{k}(u) \leq \alpha_{k}(u)$. From Lemma 2.2, we have

$$
\gamma_{k}(u)=\max _{r_{k} \leq t \leq 1-\theta_{k}} u(t) \geq \inf _{0 \leq t \leq 1} u(t) \geq \lambda(t)\|u\| \text {, so }\|u\| \leq \lambda^{-1} \gamma_{k}(u)
$$

First, we choose $u \in \partial K\left(\gamma_{k}, c_{k}\right)$, then we have $\gamma_{k}(u)=\max _{r_{k} \leq t \leq 1-\theta_{k}} u(t)=u\left(r_{k}\right)=c_{k}$. From $u(t) \leq\|u\|$ and (3.1), we can get $0 \leq u(t) \leq\|u\| \leq \lambda^{-1} u\left(r_{k}\right)=\lambda^{-1} c_{k}$, for $t \in J$. Then with $\left(H_{3}\right)$, it implies that $f(u)<\frac{c_{k}}{\rho}$, for 
$t \in J$.

$$
\text { So } \gamma_{k}(A u)=\max _{r_{k} \leq t \leq 1-\theta_{k}}(A u)(t)=A u\left(r_{k}\right) \leq(A u)(0)<\frac{c_{k}}{\rho} \frac{\int_{0}^{1} a(s) \mathrm{d} s}{1-\sum_{i=1}^{n-2} a_{i}}=c_{k}
$$

Therefore, the first condition of Lemma 1.2 satisfies.

Next, we select $u \in \partial\left(\beta_{k}, b_{k}\right)$. Then $\beta_{k}(u)=\min _{\theta_{k} \leq t \leq r_{k}} u(t)=u\left(r_{k}\right)=b_{k}$, we have $u(t) \geq b_{k}$, for $\theta_{k} \leq t \leq r_{k}$. Again from $u(t) \leq\|u\|$, and Lemma (2.2) we can get that

$$
u(t) \leq\|u\| \leq \lambda^{-1} u\left(r_{k}\right)=b_{k} / \lambda .
$$

Then $b_{k} \leq u(t) \leq b_{k} / \lambda$, for $\theta_{k} \leq t \leq r_{k}$. By $\left(H_{4}\right)$, we have $f(u)>\frac{b_{k}}{\eta}$, for $\theta_{k} \leq t \leq r_{k}$.

So, there has

$$
\begin{aligned}
\beta_{k}(A u) & =\min _{r_{k} \leq t \leq \theta_{k}}(A u)(t) \geq A u(1) \geq\left(1-\sum_{i=1}^{n-2} a_{i}\right)^{-1}\left(\sum_{i=1}^{n-2} a_{i} \int_{\xi_{i}}^{1}(1-s) a(s) f(u(s)) \mathrm{d} s\right) \\
& >\frac{b_{k}}{\eta}\left(1-\sum_{i=1}^{n-2} a_{i}\right)^{-1}\left(\sum_{i=1}^{n-2} a_{i} \int_{\xi_{i}}^{1}(1-s) a(s) \mathrm{d} s\right)=b_{k} .
\end{aligned}
$$

This implies the second condition of Lemma 1.2 is satisfied.

Finally, we only need to show the third condition of Lemma 1.2 is also satisfied.

We select $u(t) \equiv a_{k} / 2$, for $t \in J$. Obviously, $u(t) \in K\left(\alpha_{k}, a_{k}\right)$, hence $K\left(\alpha_{k}, a_{k}\right)$ is nonempty.

$\forall u(t) \in \partial\left(\alpha_{k}, a_{k}\right)$, we have $\alpha_{k}(u)=\max _{\theta_{k} \leq t \leq 1-\theta_{k}} u(t)=u\left(\theta_{k}\right)=a_{k}$. Also from $u(t) \leq\|u\|$ and Lemma (2.4), we can get $0 \leq u(t) \leq\|u\| \leq \lambda^{-1} u\left(\theta_{k}\right)=\lambda^{-1} a_{k}$, for $t \in J$. Then from $\left(H_{5}\right)$, we have $f(u)<\frac{a_{k}}{\rho}$.

So $\quad \alpha_{k}(A u)=\max _{\theta_{k} \leq t \leq 1-\theta_{k}}(A u)(t)=(A u)\left(\theta_{k}\right) \leq(A u)(0)<\frac{a_{k}}{\rho}\left(1-\sum_{i=1}^{n-2} a_{i}\right)^{-1} \int_{0}^{1} a(s) \mathrm{d} s=a_{k}$.

Then all the conditions of Lemma 1.2 are satisfied. From Lemma 1.2, we get the conclusion in Theorem 3.1.

\section{Example}

Now we consider an example to illustrate our results.

Example 4.1. Consider the boundary value problem

$$
\begin{gathered}
x^{\prime \prime}+a(t) f(u(t))=0, \quad t \in(0,1), \\
u^{\prime}(0)=0, \quad u(1)=\frac{1}{2} u\left(\frac{1}{2}\right),
\end{gathered}
$$

Then the BVP (4.1)-(4.2) can be regarded as a BVP of the form (1.1)-(1.2) in $E=C[0,1]$. In this situation, $a_{1}=\xi_{1}=\frac{1}{2}$.

Let $\delta=\frac{\pi^{2}}{3}-\frac{9}{4}, t^{\prime}=\frac{3}{8}, t_{i}=t^{\prime}-\sum_{k=1}^{i} \frac{1}{4(k+1)^{4}}, \quad i=1,2, \cdots$.

Consider the function $a(t):[0,1] \rightarrow[0,+\infty), a(t)=\sum_{i=1}^{\infty} a_{i}(t), t \in[0,1]$, where 


$$
a_{i}(t)= \begin{cases}\frac{1}{i^{2}\left(t_{i+1}+t_{i}\right)}, & 0 \leq t<\frac{t_{i+1}+t_{i}}{2} \\ \frac{\sqrt{2}}{\delta\left(t_{i}-t\right)^{\frac{1}{2}}}, & \frac{t_{i+1}+t_{i}}{2} \leq t<t_{i}, \\ \frac{\sqrt{2}}{\delta\left(t-t_{i}\right)^{\frac{1}{2}}}, & t_{i}<t \leq \frac{t_{i-1}+t_{i}}{2}, \\ 0, & \frac{t_{i-1}+t_{i}}{2}<t<1 .\end{cases}
$$

It is easy to know $\left(H_{2}\right)$ satisfies.

Let $\theta_{k} \in\left(t_{k}, t_{k+1}\right), \theta_{k}<r_{k}<1-\theta_{k},\left\{\theta_{k}\right\}_{k=1}^{\infty}$ be such that $\theta_{k} \in\left(t_{k}, t_{k+1}\right) \quad(k=1,2, \cdots)$, $\left\{a_{k}\right\}_{k=1}^{\infty},\left\{b_{k}\right\}_{k=1}^{\infty}$ and $\left\{c_{k}\right\}_{k=1}^{\infty}$ be such that $c_{k+1}<a_{k}<\lambda b_{k}<c_{k}$, and $\rho b_{k}<\eta c_{k} \quad(k=1,2, \cdots)$.

This with $\lambda<1, \rho>\eta$ implies that $\frac{c_{k+1}}{\lambda}<\frac{a_{k}}{\lambda}<b_{k}<\frac{c_{k}}{\lambda}, \frac{a_{k}}{\rho}<\frac{b_{k}}{\eta}<\frac{c_{k}}{\rho}, \quad(k=1,2, \cdots)$.

Let $f(u)= \begin{cases}\frac{a_{k}}{2 \rho}, & 0 \leq u(t) \leq \frac{a_{k}}{\lambda}, \\ \frac{1}{2}\left[\frac{a_{k}}{\rho}+\frac{\left(u(t)-\lambda^{-1} a_{k}\right)\left(\frac{b_{k}}{\eta}+\frac{c_{k}-a_{k}}{\rho}\right)}{\left(b_{k}-\lambda^{-1} a_{k}\right)}\right], & \frac{a_{k}}{\lambda}<u(t) \leq b_{k}, \\ \frac{1}{2}\left(\frac{b_{k}}{\eta}+\frac{c_{k}}{\rho}\right), & u(t)>b_{k} .\end{cases}$

Obviously, $\left(H_{3}\right),\left(H_{4}\right),\left(H_{5}\right)$ are satisfied, and it is easy to prove that $\left(H_{1}\right)$ is also satisfied. So all the conditions of Theorem 3.1 are satisfied, thus the BVP (4.1)-(4.2) has at least three infinite families of positive solutions $\left\{u_{1 k}\right\}_{k=1}^{\infty},\left\{u_{2 k}\right\}_{k=1}^{\infty}$ and $\left\{u_{3 k}\right\}_{k=1}^{\infty}$ satisfying

$$
0 \leq \alpha_{k}\left(u_{1 k}\right)<a_{k}<\alpha_{k}\left(u_{2 k}\right), \quad \beta_{k}\left(u_{2 k}\right)<b_{k}<\beta_{k}\left(u_{3 k}\right), \quad \gamma_{k}\left(u_{3 k}\right)<c_{k} \text {, for } k \in N .
$$

\section{References}

[1] Ma, R. (1999) Positive Solutions for a Nonlinear Three-Point Boundary Value Problem. Electronic Journal of Differential Equations, 34, 1-8.

[2] Liu, B. (2004) Positive Solutions of Nonlinear Four-Point Boundary Value Problem. Applied Mathematics and Computation, 155, 179-203. http://dx.doi.org/10.1016/S0096-3003(03)00770-7

[3] Chen, S.H., Hu, T., Chen, L., et al. (2005) Existence Results for $n$-Point Boundary Value Problem of Second Order Ordinary Differential Equations. Journal of Computational and Applied Mathematics, 180, 425-432. http://dx.doi.org/10.1016/j.cam.2004.11.010

[4] Ma, R. (2001) Existence of Solutions of Nonlinear m-Point Boundary Value Problem. Journal of Mathematical Analysis and Applications, 256, 556-567. http://dx.doi.org/10.1006/jmaa.2000.7320

[5] Liang, S.H. and Zhang, J.H. (2008) The Existence of Countably Many Positive Solutions for One-Dimensional p-Laplacian with Infinitely Many Singularities on the Half-Line. Applied Mathematics and Computation, 201, $210-220$. http://dx.doi.org/10.1016/j.amc.2007.12.016

[6] Xu, C., He, X. and Li, P. (2011) Global Existence of Periodic Solutions in a Six-Neuron BAM Neural Network Model 
with Discrete Delays. Neurocomputing, 74, 3257-3267. http://dx.doi.org/10.1016/j.neucom.2011.05.007

[7] Ren, J., Ge, W. and Ren, X. (2005) Existence of Positive Solutions for Quasi-Linear Boundary Value Problems. Acta Mathematicae Applicatae Sinica, 21, 353-358. (in Chinese) http://dx.doi.org/10.1007/s10255-005-0242-y 
Scientific Research Publishing (SCIRP) is one of the largest Open Access journal publishers. It is currently publishing more than 200 open access, online, peer-reviewed journals covering a wide range of academic disciplines. SCIRP serves the worldwide academic communities and contributes to the progress and application of science with its publication.

Other selected journals from SCIRP are listed as below. Submit your manuscript to us via either submit@scirp.org or Online Submission Portal.
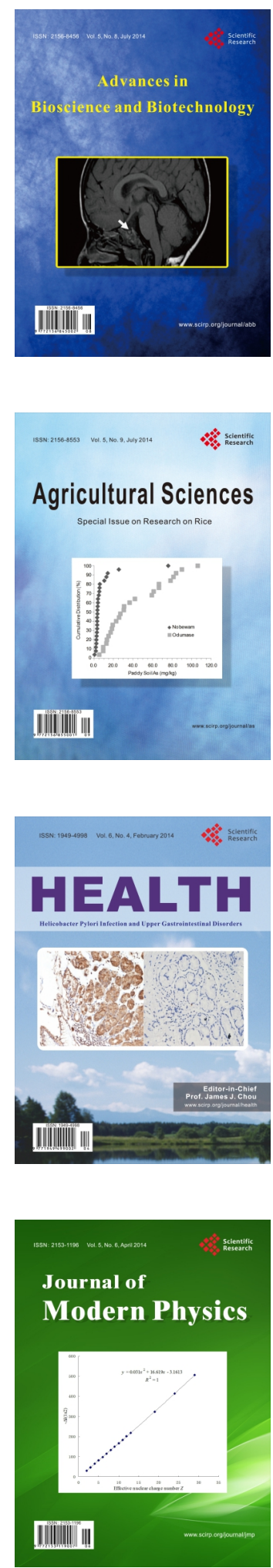
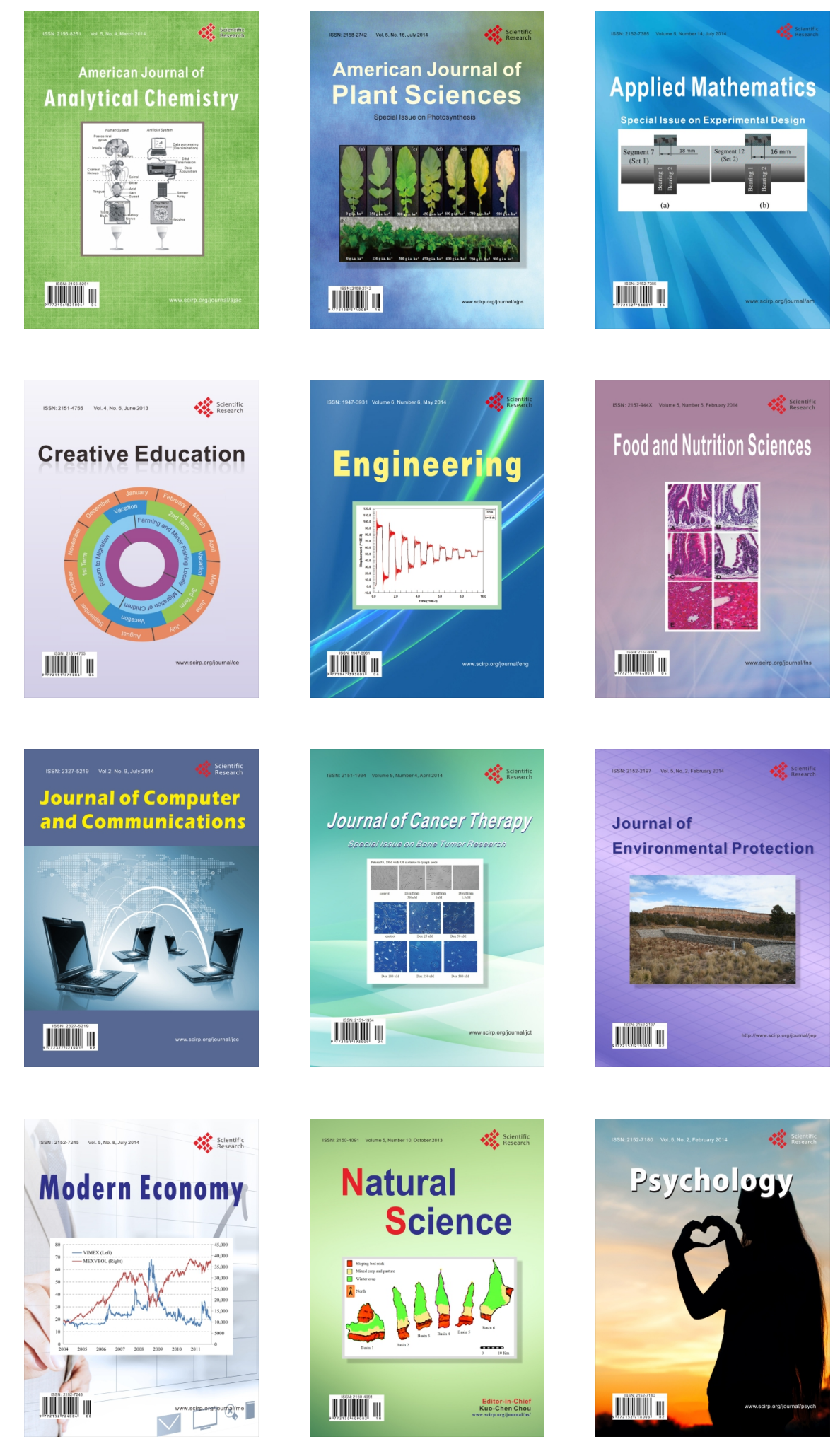